\title{
Molecular and Cellular Mechanisms of Shigella flexneri Dissemination
}

\author{
Hervé Agaisse * \\ Department of Microbiology, Immunology, and Cancer Biology, University of Virginia School of Medicine, Charlottesville, VA, \\ USA
}

The intracellular pathogen Shigella flexneri is the causative agent of bacillary dysentery in humans. The disease is characterized by bacterial invasion of intestinal cells, dissemination within the colonic epithelium through direct spread from cell to cell, and massive inflammation of the intestinal mucosa. Here, we review the mechanisms supporting S. flexneri dissemination. The dissemination process primarily relies on actin assembly at the bacterial pole, which propels the pathogen throughout the cytosol of primary infected cells. Polar actin assembly is supported by polar expression of the bacterial autotransporter family member IcsA, which recruits the N-WASP/ARP2/3 actin assembly machinery. As motile bacteria encounter cell-cell contacts, they form plasma membrane protrusions that project into adjacent cells. In addition to the ARP2/3-dependent actin assembly machinery, protrusion formation relies on formins and myosins. The resolution of protrusions into vacuoles occurs through the collapse of the protrusion neck, leading to the formation of an intermediate membrane-bound compartment termed vacuole-like protrusions (VLPS). VLP formation requires tyrosine kinase and phosphoinositide signaling in protrusions, which relies on the integrity of the bacterial type 3 secretion system (T3SS). The T3SS is also required for

OPEN ACCESS

Edited by:

Guy T. V. Nhieu,

Collège de France, France

Reviewed by:

Agathe Subtil,

Pasteur Institute, France

Kenneth G. Campellone,

University of Connecticut, USA

*Correspondence:

Hervé Agaisse

hfa5y@virginia.edu

Received: 15 December 2015 Accepted: 26 February 2016

Published: 11 March 2016

Citation:

Agaisse H (2016) Molecular and Cellular Mechanisms of Shigella flexneri Dissemination.

Front. Cell. Infect. Microbiol. 6:29. doi: 10.3389/fcimb.2016.00029 escaping double membrane vacuoles through the activity of the T3SS translocases IpaB and IpaC, and the effector proteins VirA and IcsB. Numerous factors supporting envelope biogenesis contribute to IcsA exposure and maintenance at the bacterial pole, including LPS synthesis, membrane proteases, and periplasmic chaperones. Although less characterized, the assembly and function of the T3SS in the context of bacterial dissemination also relies on factors supporting envelope biogenesis. Finally, the dissemination process requires the adaptation of the pathogen to various cellular compartments through transcriptional and post-transcriptional mechanisms.

Keywords: S. flexneri, spread from cell to cell, membrane protrusion, double membrane vacuole, IcsA, ARP2/3, $\mathrm{N}$-WASP, type 3 secretion system

\section{INTRODUCTION}

The intracellular pathogen Shigella flexneri is the causative agent of bacillary dysentery in humans (Musher and Musher, 2004). The disease is characterized by bacterial invasion of intestinal cells, dissemination within the colonic epithelium through bacterial spread from cell to cell, and massive inflammation leading to destruction of the epithelial mucosa (Sansonetti, 1998). Spreading defective but otherwise fully invasive bacterial strains are essentially avirulent, highlighting the 
ability of the bacteria to spread from cell to cell as a central determinant of S. flexneri pathogenesis (Makino et al., 1986; Bernardini et al., 1989). The spreading process relies on the acquisition of actin-based motility in the cytosol of primary infected cells (Figure 1, step 1). As motile bacteria encounter cell-cell contacts, they form plasma membrane protrusions that project into adjacent cells (Figure 1, step 2). The formed membrane protrusions then transition into an intermediate membrane-bound compartment termed vacuole-like protrusions (VLP) (Figure 1, step 3), whose resolution leads to the formation of genuine vacuoles (Figure 1, step 4). S. flexneri subsequently escapes from the formed double membrane vacuoles (Figure 1, step 5), thereby gaining access to the cytosol of adjacent cells and achieving cell-to-cell spread (Figure 1, step 6). The reiteration of this spreading process supports the propagation of the pathogen within the intestinal epithelium. The formation of membrane protrusions and double membrane vacuoles during infection was first documented in electron microscopy studies using rhesus monkeys as animal models of S. flexneri infection (Takeuchi et al., 1968). Further electron microscopy studies in human epithelial cell lines confirmed the formation of protrusions and vacuoles in infected cells, providing suitable in vitro systems for investigating the mechanisms supporting $S$. flexneri dissemination (Kadurugamuwa et al., 1991; Gouin et al., 1999). The efficiency of S. flexneri dissemination is commonly assessed by using plaque formation as readout of cell-to-cell spread (Oaks et al., 1985). Recently, live fluorescence microscopy was used to capture and quantify the whole sequence of events occurring as single bacteria spread from cell to cell through protrusion and vacuole formation (Dragoi and Agaisse, 2014, 2015). Here, we review three decades of research on the mechanisms supporting S. flexneri dissemination.

\section{ACTIN-BASED MOTILITY IN S. FLEXNERI DISSEMINATION}

S. flexneri acquires intracellular motility in the cytosol of primary infected cells through manipulation of the host cell actin cytoskeleton (Welch and Way, 2013). The assembly and expansion of the actin network at the bacterial pole propels the pathogen throughout the cytosolic compartment, and leads to the formation of filamentous actin structures referred to as actin tails (Vasselon et al., 1992). Seminal genetic studies led to the identification of the bacterial factor IcsA (aka VirG) as the major determinant of $S$. flexneri actin-based motility (Makino et al., 1986; Bernardini et al., 1989). IcsA is a member of the autotransporter family of proteins, which display a carboxy-terminal beta domain that forms a beta barrel channel and mediates the transfer of the (passenger) amino-terminal alpha domain across the outer membrane (Henderson and Nataro, 2005). The exposure of the passenger domain of IcsA to the cytosol leads to the recruitment of the nucleation-promoting factor N-WASP (Figure 2, step 1; Suzuki et al., 1998; Egile et al., 1999). In turn, NWASP recruits and activates a major actin nucleator, the ARP2/3 complex (Figure 2, step 1; Egile et al., 1999; Loisel et al., 1999). In cells, the activity of N-WASP is usually regulated by the

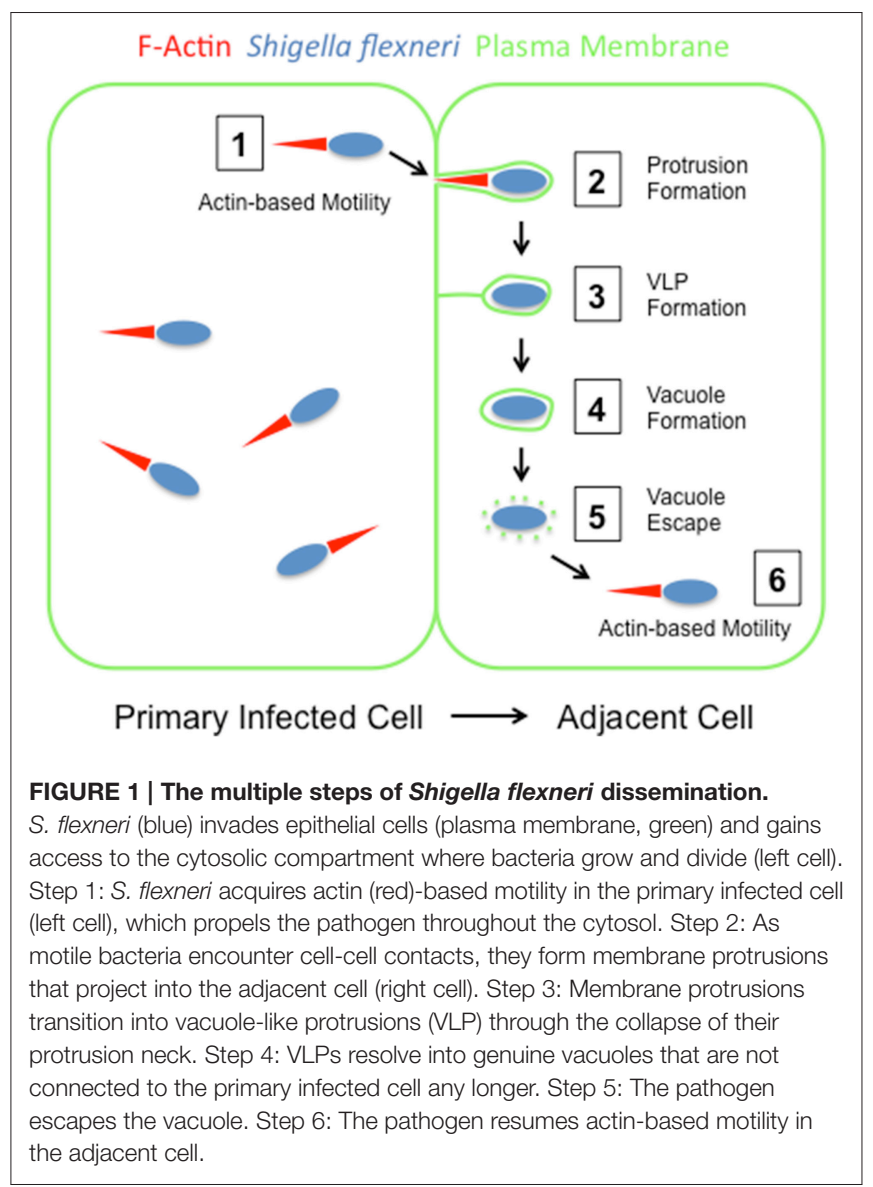

small-GTPase Cdc42 (Rohatgi et al., 1999). However, N-WASP recruitment and actin tail assembly at the bacterial pole do not require Cdc42 (Mounier et al., 1999; Shibata et al., 2002). The minimal region of IcsA required for N-WASP recruitment and actin polymerization in vitro resides in the passenger domain of IcsA (amino acid residues 56-508; Suzuki et al., 1996). Interestingly, IcsA interacts specifically with N-WASP, but not with other N-WASP/WAVE family members, such as WASP (Suzuki et al., 2002), which explains why S. flexneri displays actinbased motility in N-WASP-expressing cells, such as epithelial cells, but not in WASP-expressing cells, such as macrophages. Chimera experiments using WASP and N-WASP revealed that $\mathrm{N}$-WASP binds IcsA through its GTPase-binding domain that normally binds Cdc42 (Suzuki et al., 2002). Altogether, these studies suggest that IcsA acts as a functional mimic of Cdc42, releasing N-WASP from its auto-inhibitory conformation and facilitating the interaction of the nucleation-promoting domain (VCA) of N-WASP with the ARP2/3 complex (Kim et al., 2000). In addition to N-WASP, the passenger domain of IcsA has been shown to interact with IcsB (Ogawa et al., 2005), a T3SS effector protein required for $S$. flexneri dissemination (Allaoui et al., 1992; Ogawa et al., 2003). The exact function of IcsB is however complex and spans from inhibition of autophagy to disruption of the double membrane vacuole (Ogawa et al., 2005; Campbell-Valois et al., 2015). The exact significance of the 


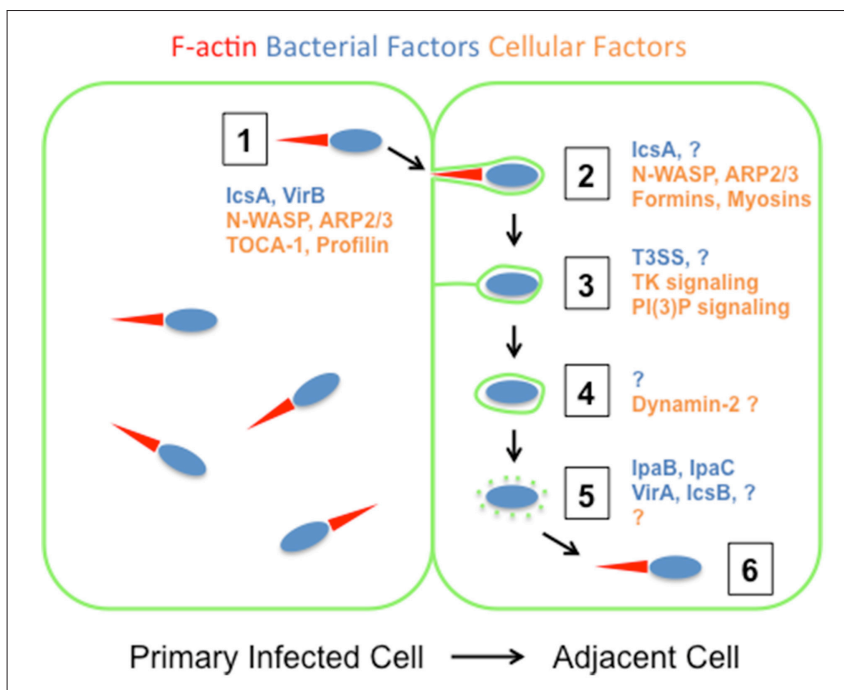

FIGURE 2 | Bacterial and cellular factors supporting Shigella flexneri dissemination. The bacterial and cellular factors involved in S. flexneri dissemination are depicted in blue and orange, respectively. Green lines indicate the plasma membrane. Step 1: actin-based motility relies on IcsA that recruits N-WASP, thereby leading to ARP2/3-dependent actin nucleation (red) at the bacterial pole. N-WASP activation is also supported by the VirB-dependent recruitment of TOCA-1. Additional cytoskeleton regulators, such as Profilin stimulate actin-based motility. Step 2: the formation of membrane protrusions is most likely supported by the factors supporting cytosolic motility, including IcsA, N-WASP and the ARP2/3 complex. In addition, formins and myosins are required for efficient protrusion formation. Step 3: membrane protrusions transition into vacuole-like protrusions through a process that rely on the type 3 secretion system (T3SS)-dependent activation of tyrosine kinase and phosphoinositide signaling. Step 4: VLPS transition into genuine vacuoles through resolution of the protrusion neck, a process that potentially relies on the activity of the Dynamin 2-dependent membrane scission machinery in the adjacent cell (right cell). Step 5: the pathogen escapes from the vacuole through a mechanism that requires the activity of the T3SS translocases IpaB and IpaC, as well as the effector protein VirA and IcsB. Step 6: the pathogen resumes actin-based motility through the mechanisms depicted in Step 1.

IcsB-IcsA interaction in the context of $S$. flexneri dissemination remains therefore to be clarified. The passenger domain of IcsA was also shown to display potential sites for phosphorylation by cyclic AMP-dependent kinases (amino acid residues 754760). The introduction of mutations preventing phosphorylation increased cell-to-cell spread, but only during the first $3 \mathrm{~h}$ of infection (d'Hauteville and Sansonetti, 1992).

In order to trigger N-WASP activation, S. flexneri also recruits to the bacterial surface the transducer of Cdc42-dependent actin assembly, TOCA-1 (Figure 2, step 1; Leung et al., 2008). TOCA-1 is member of the F-BAR family of membrane-deforming proteins that contribute to N-WASP activation (Ho et al., 2004). As opposed to N-WASP, the recruitment of TOCA-1 does not rely on IcsA, but requires the integrity of the transcriptional regulator VirB. This suggests a role for VirB-regulated virulence factors, including the T3SS, in TOCA-1 recruitment and further extend the notion that $S$. flexneri evolved complex functional mimicry in order to hijack the N-WASP/TOCA-1 machinery (Leung et al., 2008). N-WASP activation in the context of S. flexneri actin tail formation also relies on N-WASP tyrosine phosphorylation, which is positively regulated by the tyrosine kinases $\mathrm{Abl}$ and Btk, in fibroblasts and intestinal epithelial cells, respectively (Burton et al., 2005; Dragoi et al., 2013). In addition to the requirement for N-WASP and TOCA-1, biochemical and genetic studies indicated that additional cytoskeleton regulators, such as profilin, stimulate $S$. flexneri actin-based motility in vitro as well as in vivo (Loisel et al., 1999; Mimuro et al., 2000). Finally, N-WASP phosphorylation was shown to increase in a Btk-dependent manner in response to bacterial infection, suggesting that in addition to the components of the actin assembly machinery, $S$. flexneri manipulates signaling events that contribute to the efficiency of actin assembly at the bacterial pole, through unknown mechanisms (Dragoi et al., 2013).

\section{PROTRUSION FORMATION IN S. FLEXNERI DISSEMINATION}

As bacteria displaying cytosolic motility reach the cell periphery, they deform the plasma membrane and form membrane protrusions. In order to lead to efficient cell-to-cell spread, the formed protrusions must project into adjacent cells (Figure 1, step 2). This is facilitated by the formation of E-cadherindependent cell-cell contacts in epithelial cells (Sansonetti et al., 1994). It was also suggested that tricellular junctions represent preferred sites of protrusion formation at the plasma membrane (Fukumatsu et al., 2012). In addition to structural components mediating cell-cell contacts, bacterial dissemination is also supported by connexin-dependent signaling events at the plasma membrane, through the release of signaling molecules, such as ATP (Tran Van Nhieu et al., 2003). There is also evidence for an active role played by the pathogen in the maintenance of cell-cell contacts supporting dissemination. This is exemplified by the observation that a mutant strain defective in the expression of OspE2, a type 3 secretion effector protein injected into host cells, leads to premature rounding up of infected cells, a reflection of cell-cell contact disruption (Miura et al., 2006; Kim et al., 2009). In order to form membrane protrusions, S. flexneri utilizes the forces developed by the actin cytoskeleton to deform the plasma membrane and project into adjacent cells. Similar to the mechanisms supporting actin tail formation in the cytosol, it is likely that actin polymerization in membrane protrusions relies on IcsA and the N-WASP/ARP2/3-dependent machinery (Figure 2, step 2 ). However, investigating the role of IcsA, N-WASP, and the ARP2/3 complex specifically in protrusions is difficult because disruption of the activity of these factors would primarily affect actin-based motility in the cytosol, and therefore prevent protrusions formation. Interestingly, a genetic screen for peptide insertions that interfere with IcsA function identified several positions (amino-acid residues 56, 193, 288, 312, and 502) at which peptide insertion hardly affected actin tail formation in the cytosol, and yet strongly affected plaque formation (May and Morona, 2008). The corresponding positions map to the region required for $\mathrm{N}$-WASP recruitment and may be involved in the recruitment of host factors specifically required for 
protrusion formation, through a mechanism that remain to be further explored. In addition to the ARP2/3 complex, recent studies suggested the involvement of protrusions-specific and ARP2/3-independent actin nucleators in protrusion formation. In particular, Diaphanous-related formins have been identified as important determinants of protrusion formation in S. flexneri infected cells (Figure 2, step 2) (Heindl et al., 2010). Whether these formins contribute to actin polymerization at the bacterial pole, in concert with the ARP2/3 complex, or display additional functions in protrusions, needs to be further investigated. Future research should also examine whether similar to IcsA and the recruitment of the N-WASP/ARP2/3 machinery, pathogenspecific features regulate the recruitment/activity of formins in $S$. flexneri protrusions. In addition to cellular components supporting actin assembly, such as the ARP2/3 complex and formins, additional cytoskeleton factors contribute to protrusion formation, including myosins (Figure 2, step 2). Myosin X was recently found to localize to the plasma membrane surrounding S. flexneri protrusions and move along the bacterial sides (Bishai et al., 2013). Myosin X depletion resulted in the formation of shorter and wider protrusions, indicating a role for this protein in proper protrusion formation (Bishai et al., 2013). Structure function analyses suggested that Myosin X contributes to $S$. flexneri protrusion formation by bridging the actin cytoskeleton and the plasma membrane, and potentially transporting unknown cargoes within protrusions (Bishai et al., 2013). S. flexneri dissemination also relies on Myosin II and its regulator, the myosin light chain kinase MLCK (Rathman et al., 2000a; Lum and Morona, 2014). Interestingly, Myosin II is not required for the formation of Listeria monocytogenes protrusions (Rathman et al., 2000a), indicating a specific function for the Myosin II pathway in S. flexneri dissemination, which remains to be determined.

\section{PROTRUSION RESOLUTION IN S. FLEXNERI DISSEMINATION}

Recent live confocal microscopy of intestinal cells infected with $S$. flexneri revealed the formation of membrane protrusions that first undergo elongation and then transitioned into a membrane-bound compartment referred to as vacuole-like protrusions (Figure 1, step 3, VLP; Dragoi and Agaisse, 2015). VLPs display a continuous lining of the plasma membrane around the bacterium, but remain connected to the primary infected cells through a membranous tether. The tether results from the complete collapse of the protrusion neck, which likely reflects the collapse of the underlying actin cytoskeleton (Dragoi and Agaisse, 2014). The plasma membrane surrounding the protrusions formed by $S$. flexneri is highly enriched in phosphorylated tyrosine residues, suggesting a role for tyrosine kinase signaling in protrusions (Dragoi and Agaisse, 2014). Accordingly, treatment with the tyrosine kinase inhibitor Gleevec inhibited S. flexneri dissemination (Dragoi and Agaisse, 2014). Interestingly, tyrosine kinase signaling is supported by the cell polarity serine/threonine kinase STK11 through a mechanism that may involve the proper trafficking of receptor tyrosine kinases to cell-cell contacts (Dragoi and Agaisse, 2014). In addition to tyrosine phosphorylation, $S$. flexneri dissemination through protrusion collapse and VLP formation also requires the production of phosphatidylinositol3-phosphate $(\mathrm{PI}(3) \mathrm{P})$ in protrusions, which relies on the class II phosphatidylinositol-3 kinase PIK3C2A (Dragoi and Agaisse, 2015). Interestingly, Listeria monocytogenes spread from cell to cell did not rely on tyrosine kinase and phosphoinositide signaling (Dragoi and Agaisse, 2015), highlighting the notion that S. flexneri and L. monocytogenes have evolved pathogenspecific mechanisms of bacterial dissemination (Kuehl et al., 2015). The first evidence of a role for the bacterial T3SS in the resolution of $S$. flexneri protrusions into vacuoles came from the isolation of a transposon in the ipgC gene, which encodes a molecular chaperone for IpaB and IpaC (Rathman et al., 2000b). The corresponding ipgC mutant grew normally in the cytosol of infected cells, where it displayed actinbased motility, but failed to spread from cell to cell. Electron microscopy studies revealed that the ipgC mutant resided in protrusions displaying several layers of membrane, as if the protrusions kept elongating and circling at sites of cell-cell contacts, instead of transitioning into vacuoles (Rathman et al., 2000b). Confirming the notion that the T3SS is required for proper dissemination, two independent studies demonstrated a role for MxiG, a structural component of the T3SS, in the resolution of protrusion into vacuoles (Allaoui et al., 1995; Kuehl et al., 2014). Moreover, MxiG was shown to be required for activation of tyrosine kinase signaling in $S$. flexneri protrusions (Kuehl et al., 2014). Altogether, these observations thus suggest that $S$. flexneri utilizes the T3SS to manipulate tyrosine kinase signaling and downstream signaling events, such as $\mathrm{PI}(3) \mathrm{P}$ production, in order to mediate the collapse of the actin cytoskeleton in protrusions, which leads to VLP formation. Although the involvement of the T3SS in S. flexneri dissemination has been clearly established, the identity of the effector proteins potentially required for the manipulation of the signaling events observed in protrusions remains to be determined.

The mechanisms supporting the transition of VLPs into vacuoles are poorly understood (Figure 2, step 4). Live imaging studies suggested that the formation of genuine vacuoles occurs through the gradual disappearance of the membrane tether connecting VLPs to the primary infected cells (Dragoi and Agaisse, 2015). Interestingly, genetic depletion and pharmacological inhibition suggested a role for the Dynamin 2-dependent membrane scission machinery in $S$. flexneri dissemination (Fukumatsu et al., 2012). The resolution of the VLP membrane tether leading to vacuole formation may thus rely on the activity of Dynamin 2 in adjacent cells (Figure 2, step 4). Together with Dynamin 2, the class I PI3K, clathrin, epsin1 and dynamin-2, but not AP-2, Dab2, and Eps15, were shown to be required for $S$. flexneri dissemination. These observations led the authors to propose a model in which the uptake of protrusions by adjacent cells occurs through a non-canonical form of endocytosis. Although clathrin has been shown to be recruited to large objects such as bacteria and be required for their uptake (Veiga and Cossart, 2006), it remains unclear how 
the known function of clathrin in coating small vesicles may assist in the internalization of large objects, including S. flexneri protrusions. Future research is therefore needed to clarify the role of Dynamin 2 and clathrin-mediated endocytosis in S. flexneri dissemination.

\section{VACUOLE ESCAPE IN S. FLEXNERI DISSEMINATION}

The use of inducible systems to conditionally express components of the T3SS demonstrated that, in addition to its role in protrusions, the T3SS is also necessary for efficient escape from vacuoles (Figure 2, step 5; Page et al., 1999; Schuch et al., 1999; Kuehl et al., 2014). Electron microscopy studies first suggested that T3SS-defective mutants accumulate in the double membrane vacuoles formed during the dissemination process (Page et al., 1999; Schuch et al., 1999). These observations were recently confirmed by live imaging microscopy studies in which the tracking individual bacteria provided the unambiguous demonstration that a conditional mutant defective in the expression of the T3SS could not escape the vacuoles deriving from protrusion resolution (Kuehl et al., 2014). Interestingly, the use of a transcriptional reporter system to monitor the activity of the T3SS showed that T3 secretion is activated upon invasion, shut down in the cytosol, and re-activated in protrusions and vacuoles (Campbell-Valois et al., 2014). Moreover, the use of T3SS activity reporter in combination with bacterial mutants suggested that the VirA and IcsB mutants accumulate in double membrane vacuoles (Figure 2, step 5; Campbell-Valois et al., 2015). The function of IcsB is unknown, but several activities have been attributed to VirA, including inhibition of the small-GTPase Rab1 (Dong et al., 2012), and activation of the Calpain system (Bergounioux et al., 2012), through an unknown mechanism. The exact role of VirA in vacuole escape remains to be determined. As the single VirA and IcsB mutants hardly displayed plaque formation defects, and the double mutant did form smaller plaques (Campbell-Valois et al., 2015), it is likely that cooperative activities provided by various effector proteins contribute to double membrane vacuole escape. This may include the phosphoinositide phosphatase IpgD, which was shown to contribute to the escape from the primary vacuoles formed upon invasion (Mellouk et al., 2014). In addition to defining the complete set of effectors contributing to vacuole escape, the elucidation of the vacuole escape mechanisms will require the identification of the cellular components targeted by the T3SS effector proteins (Figure 2, step 5). Interestingly, a recent report established a link between the autophagy machinery and the repair of membrane damage caused by the activity of the T3SS in the primary vacuoles formed upon Salmonella infection (Kreibich et al., 2015). Given the proposed role of IcsB and VirA in countering autophagy (Ogawa et al., 2005; Dong et al., 2012; Campbell-Valois et al., 2015), it is tempting to speculate that vacuole escape partly occurs as a result of the failure of the autophagy machinery to repair the membrane of the vacuoles formed upon S. flexneri infection.

\section{BACTERIAL ENVELOPE BIOGENESIS IN S. FLEXNERI DISSEMINATION}

S. flexneri dissemination relies on IcsA and the T3SS, two components of the bacterial envelope whose proper exposure on the bacterial surface is critical for their function. Not surprisingly, various determinants of bacterial envelope biogenesis have thus been identified as essential determinants of $S$. flexneri dissemination (Table 1).

\section{Establishment of IcsA Polarity}

The formation of actin tails at the bacterial pole relies on the establishment and maintenance of IcsA polarity (Goldberg et al., 1993). Although IcsA secretion relies on the circumferentially expressed Sec machinery, IcsA is only secreted at the bacterial pole (Brandon et al., 2003). Two regions in IcsA, termed region 1 (amino acid residues 56-104) and region 2 (amino acid residues 507-620) contribute to the polar localization of IcsA in the cytosol, prior to secretion (Charles et al., 2001). Genetic screens uncovered DnaK and FtsQ as regulators of IcsA cytoplasmic chaperoning and positioning at the bacterial poles, respectively (Fixen et al., 2012). In the periplasm, the protease/chaperone DegP functions in partnership with the chaperones Skp and SurA and mediates full exposure of the alpha domain of IcsA at the bacterial surface (Purdy et al., 2007).

\section{Maintenance of ICSA Polarity}

Early observations pointed to a role for LPS in S. flexneri dissemination (Okamura and Nakaya, 1977) and numerous genetic studies subsequently demonstrated the essential role of short $\mathrm{O}$ antigen assembly in plaque formation (Table 1). LPS-defective mutants are not able to form polar actin tails and instead polymerize actin circumferentially on the bacterial surface (Rajakumar et al., 1994; Sandlin et al., 1995; Van den Bosch et al., 1997). Interestingly, IcsA localized circumferentially in O antigen defective strains (Sandlin et al., 1995), suggesting that LPS supports the maintenance of IcsA at the bacterial pole by restricting its lateral diffusion. In addition to LPS, the serine protease IcsP was proposed to contribute to the maintenance of IcsA polarity by releasing the $\mathrm{N}$-terminal domain from the bacterial sides (Shere et al., 1997). However, compared to wildtype bacteria, the ics $P$ mutant was indeed more efficient at moving in the cytosol and spreading from cell to cell, indicating that the IcsP-mediated cleavage of IcsA is in fact detrimental to S. flexneri dissemination (Shere et al., 1997). It is noteworthy that outer membrane proteins detrimental to IcsA expression in E. coli, such as OmpT, are no longer encoded in S. flexneri spp. genomes (Nakata et al., 1993). Deletion of "anti-virulence" genes has been proposed to be one of the major determinants in the evolution of S. flexneri pathogenic properties, including the acquisition of IcsA-mediated actin-based motility (Bliven and Maurelli, 2012).

\section{Assembly of the T3SS}

$\mathrm{VpsC}$ was identified as a determinant of $S$. flexneri dissemination in a screen for bacterial factors required for plaque formation (Hong et al., 1998). VpsC is part of the Vps/VacJ transporter 
TABLE 1 | The bacterial genes supporting Shigella flexneri dissemination.

\begin{tabular}{|c|c|c|c|}
\hline Gene & Function & Defect & References \\
\hline$i c s A$ & N-WASP recruitment & Actin-based motility & Makino et al., 1986; Bernardini et al., 1989 \\
\hline$r f b B C A D$ & LPS biosynthesis & IcsA polarity & Rajakumar et al., 1994 \\
\hline gall & LPS biosynthesis & IcsA polarity & Sandlin et al., 1995 \\
\hline rfe & LPS biosynthesis & IcsA polarity & Sandlin et al., 1995 \\
\hline rol & LPS biosynthesis & IcsA polarity & Morona et al., 1995 \\
\hline ompt & Protease & IcsA exposure & Nakata et al., 1993 \\
\hline ompA & $?$ & IcsA exposure & Ambrosi et al., 2012 \\
\hline $\operatorname{deg} P$ & Protease/Chaperone & IcsA exposure & Purdy et al., 2002 \\
\hline skp & Chaperone & IcsA exposure & Purdy et al., 2007 \\
\hline surA & Chaperone & IcsA exposure & Purdy et al., 2007 \\
\hline phoN2 & Apyrase & IcsA exposure & Santapaola et al., 2006 \\
\hline ompA & $?$ & IcsA exposure & Scribano et al., 2014 \\
\hline ispA & $?$ & Septation & Mac Síomóin et al., 1996 \\
\hline virk & $?$ & Envelope Biogenesis & Nakata et al., 1992; Sidik et al., 2014 \\
\hline vpsC & ABC Transporter & Envelope Biogenesis & Hong et al., 1998 \\
\hline vacJ & ABC Transporter & Envelope Biogenesis & Suzuki et al., 1994; Carpenter et al., 2014 \\
\hline$d s b A$ & Periplasmic oxidoreductase & IpaB/IpaC secretion Protrusion resolution & Watarai et al., 1995; Yu et al., 2000 \\
\hline$d k s A$ & Transcription Factor & Hfq expression & Mogull et al., 2001 \\
\hline$h f q$ & RNA binding & VirB expression? & Sharma and Payne, 2006 \\
\hline pstS & Phosphate binding & PhoB regulon mis-expression & Runyen-Janecky and Payne, 2002 \\
\hline $\begin{array}{l}\text { ipaB } \\
\text { ipaC }\end{array}$ & T3SS Translocases & Vacuole escape & Page et al., 1999; Schuch et al., 1999 \\
\hline ipgC & T3SS Chaperone & Protrusion resolution Vacuole escape? & Rathman et al., 2000b \\
\hline$m \times i E$ & Transcription Factor & Effector protein expression & Kane et al., 2002 \\
\hline$m \times i G$ & Structural component & Protrusion resolution Vacuole escape & Allaoui et al., 1995; Kuehl et al., 2014 \\
\hline virA & Rab1 GAP, Calpain activation & Vacuole escape & Uchiya et al., 1995; Dong et al., 2012; Campbell-Valois et al., 2015 \\
\hline$i c s B$ & $?$ & Vacuole escape & Allaoui et al., 1992; Ogawa et al., 2003, 2005; Campbell-Valois et al., 2015 \\
\hline
\end{tabular}

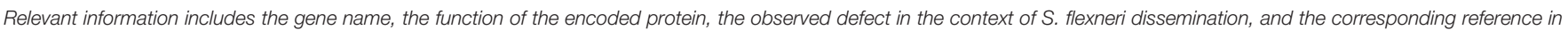
the cited literature.

system and a similar plaque formation defect was originally observed for the vacJ mutant (Suzuki et al., 1994). The $\mathrm{Vps} / \mathrm{VacJ}$ system has been proposed to regulate the levels of phospholipids in the outer leaflet of the outer membrane in E. coli (Malinverni and Silhavy, 2009). However, enzymatic manipulation of phospholipid composition aiming at restoring normal levels of phospholipids in the outer membrane, failed to correct the plaque formation defect observed with the $v p s C$ mutant (Hong et al., 1998). Interestingly, the $\mathrm{VpsC}$ mutant secretes elevated levels of the virulence factors IcsA, IpaB, IpaC, and IpaD (Hong et al., 1998) and a similar hypersecretion phenotype was reported recently for the virK mutant (Sidik et al., 2014). The exact function of VirK is unknown, but hypersecretion is apparently due to an increase in the release of outer membrane vesicles (OMVs). Moreover, a similar increase in OMV release was reported for a mutant in the periplasmic chaperone DegP. As both virK and $\operatorname{deg} P$ showed genetic interactions with $m x i D$, a structural component of T3SS, it was proposed that VirK and DegP may relieve the periplasmic stress associated with the assembly of the T3SS (Sidik et al., 2014). Also required for the proper assembly of the T3SS is the periplasmic thiol:disulfide oxidoreductase DsbA. Interestingly, the $d b s A$ mutant displayed normal actin-based motility, but failed to resolve membrane protrusions into vacuoles ( $\mathrm{Yu}$ et al., 2000). This defect was associated with defective secretion of the translocases, IpaB and IpaC, in protrusions (Watarai et al., 1995; Yu et al., 2000). Since the $d b s A$ mutant did not display any T3SS-dependent invasion defects, it is likely that the activity of DbsA is specifically required for the assembly of the T3SS in protrusions. These observations point to the fact that we know very little about the mechanisms supporting the post-invasion assembly of the T3SS in various cellular compartments, including the cytosol, protrusions and vacuoles.

\section{BACTERIAL ADAPTATION IN S. FLEXNERI DISSEMINATION}

S. flexneri is an intracellular pathogen that resides in the cytosol of infected cells, but also encounters various membranebound compartments upon dissemination, including membrane protrusions and vacuoles in which the bacteria spend an average of $\sim 15$ and $\sim 30 \mathrm{~min}$, respectively (Dragoi and Agaisse, 2015). S. flexneri may thus need to adapt to various cellular environments in order to disseminate successfully. Accordingly, a screen for bacterial genes induced in the intracellular environment led to the identification of genes required for phosphate acquisition 
(pstS), sugar import (uphT) and genes activated in response to iron depletion (sitA; Runyen-Janecky and Payne, 2002). Although the $u p h T$ and sitA mutant strains formed normal plaques, mutants in the Pst system formed smaller plaque compared to wild type (Runyen-Janecky and Payne, 2002). The spreading defect was not related to PhoA-mediated phosphate transport, but involved the de-regulation of the $\mathrm{PhoB}$ regulon, which did not impact IcsA polarity or bacterial growth, at least during the first $3 \mathrm{~h}$ of infection (Runyen-Janecky et al., 2005). Why the expression of PhoB-regulated genes negatively impacted S. flexneri dissemination is unclear, but it indicates the importance of proper regulation of bacterial gene expression profiles in the dissemination process. In addition to the main transcriptional regulators of S. flexneri virulence, VirB and VirF, the transcriptional activator MxiE regulates the expression of a subset of T3SS effector proteins (Kane et al., 2002; Mavris et al., 2002). MxiE is regulated by the activity of the T3SS through a complex mechanism involving the chaperone IpgC, acting as MxiE co-activator, and the secreted effector OspD1, acting as MxiE inhibitor (Parsot et al., 2005). When the T3SS is not active, the interaction of OspD1 with MxiE prevents transcriptional activation of the MxiE-regulated genes. As the T3SS system gets activated in protrusions or vacuoles, probably upon insertion of the translocases into the plasma membrane, the secretion of OspD1 frees up MxiE, which triggers the transcriptional activation of MxiE-regulated genes. As the mxiE mutant displays a defect in cell-to-cell spread (Kane et al., 2002), it is likely that a set of MxiE-regulated effectors proteins, contributes to $S$. flexneri dissemination. Recent work pointed to a role for the MxiE-regulated effector protein VirA in vacuole escape (Campbell-Valois et al., 2015). Future research should therefore systematically explore the potential role of MxiEregulated effectors proteins in S. flexneri dissemination.

\section{CONCLUDING REMARKS}

In the past three decades, we have made tremendous advances in our understanding of the mechanisms supporting $S$. flexneri dissemination through cell-to-cell spread. Genetic investigations have revealed two critical bacterial determinants of dissemination: the auto-transporter IcsA and the T3SS.

The role of IcsA in acquisition of actin-based motility in the cytosol, though interaction with the N-WASP/ARP2/3 actin assembly machinery, has been extensively investigated and is now fairly well understood. By contrast, the mechanisms supporting actin assembly in membrane protrusions are still poorly understood. In particular, the exact role of cytoskeleton factors, such as formins and myosins, and potential bacterial factors, specifically required for membrane protrusion formation need further attention.

Genetic investigations have demonstrated the requirement of the T3SS for efficient S. flexneri dissemination. The determination of the exact mechanisms supporting T3SSdependent dissemination will not only require the identification and characterization of the T3SS effector proteins potentially involved, but also the identification and characterization of the cellular processes targeted by the T3SS effector proteins. This relates to T3SS-dependent actin-based motility, the complex protrusion-to-VLP-to-vacuole transition, and the escape from double membrane vacuoles. Given the multiple roles of the T3SS, different sets of effectors may act cooperatively in different cellular compartments, perhaps suggesting the existence of uncharacterized regulatory mechanisms controlling the activity of the T3SS in space and time during the course of dissemination.

Beyond the IcsA- and T3SS-dependent mechanisms mediating cytosolic motility and cell-to-cell spread, it is unclear whether specific mechanisms support bacterial fitness in the various cellular compartments encountered by the pathogen during dissemination, including the cytosol, membrane protrusions and double membrane vacuoles. How the bacteria may sense, respond and adapt to these various cellular environments remains poorly understood and may represent critical aspects of the dissemination process. Finally, although the dissemination process is central to the extensive damage inflicted to the intestinal epithelium during infection, the exact role of cell-to-cell spread in S. flexneri pathogenesis is still unclear. It is generally admitted that the destruction of the epithelial mucosa results from the infiltration of immune cells in response to pro-inflammatory molecules produced by infected epithelial cells. Although the dissemination process certainly contributes to the magnitude of the inflammatory response by enlarging the numbers of epithelial cells producing pro-inflammatory molecules, the impact of cell-to-cell spread on epithelial homeostasis may be far more complex than currently understood.

In conclusion, three decades of research on $S$. flexneri actin-based motility and cell-to-cell spread have contributed to our current understanding of the molecular and cellular mechanism supporting $S$. flexneri dissemination. It is noteworthy that, in addition to $S$. flexneri, actin-based motility and cell-to-cell spread are essential aspects of the infectious cycle of various intracellular pathogens, including Listeria monocytogenes, Rickettsia spp. and Burkholderia spp. Recent studies have highlighted the notion that, although relying on common strategies of intracellular motility based on the actin cytoskeleton, S. flexneri and L. monocytogenes have in fact evolved strikingly different mechanisms of cell-to-cell spread (Talman et al., 2014; Dragoi and Agaisse, 2015; Kuehl et al., 2015). Further comparative analyses of the mechanisms supporting S. flexneri, L. monocytogenes, Rickettsia spp. and Burkholderia spp. spread from cell to cell may thus reveal an astonishing diversity of mechanisms supporting bacterial dissemination.

\section{AUTHOR CONTRIBUTIONS}

The author confirms being the sole contributor of this work and approved it for publication.

\section{ACKNOWLEDGMENTS}

This work was supported by the National Institutes of Health grant R01AI073904 (HA). 


\section{REFERENCES}

Allaoui, A., Mounier, J., Prévost, M. C., Sansonetti, P. J., and Parsot, C. (1992). icsB: a Shigella flexneri virulence gene necessary for the lysis of protrusions during intercellular spread. Mol. Microbiol. 6, 1605-1616. doi: 10.1111/j.13652958.1992.tb00885.x

Allaoui, A., Sansonetti, P. J., Ménard, R., Barzu, S., Mounier, J., Phalipon, A., et al. (1995). MxiG, a membrane protein required for secretion of Shigella spp. Ipa invasins: involvement in entry into epithelial cells and in intercellular dissemination. Mol. Microbiol. 17, 461-470. doi: 10.1111/j.13652958.1995.mmi_17030461.x

Ambrosi, C., Pompili, M., Scribano, D., Zagaglia, C., Ripa, S., and Nicoletti, M. (2012). Outer membrane protein A (OmpA): a new player in shigella flexneri protrusion formation and inter-cellular spreading. PLoS ONE 7:e49625. doi: 10.1371/journal.pone.0049625

Bergounioux, J., Elisee, R., Prunier, A. L., Donnadieu, F., Sperandio, B., Sansonetti, P., et al. (2012). Calpain activation by the Shigella flexneri effector VirA regulates key steps in the formation and life of the bacterium's epithelial niche. Cell Host Microbe 11, 240-252. doi: 10.1016/j.chom.2012.01.013

Bernardini, M. L., Mounier, J., d'Hauteville, H., Coquis-Rondon, M., and Sansonetti, P. J. (1989). Identification of icsA, a plasmid locus of Shigella flexneri that governs bacterial intra- and intercellular spread through interaction with F-actin. Proc. Natl. Acad. Sci. U.S.A. 86, 3867-3871. doi: 10.1073/pnas.86.10.3867

Bishai, E. A., Sidhu, G. S., Li, W., Dhillon, J., Bohil, A. B., Cheney, R. E., et al. (2013). Myosin-X facilitates Shigella-induced membrane protrusions and cell-to-cell spread. Cell. Microbiol. 15, 353-367. doi: 10.1111/cmi.12051

Bliven, K. A., and Maurelli, A. T. (2012). Antivirulence genes: insights into pathogen evolution through gene loss. Infect. Immun. 80, 4061-4070. doi: 10.1128/IAI.00740-12

Brandon, L. D., Goehring, N., Janakiraman, A., Yan, A. W., Wu, T., Beckwith, J., et al. (2003). IcsA, a polarly localized autotransporter with an atypical signal peptide, uses the Sec apparatus for secretion, although the Sec apparatus is circumferentially distributed. Mol. Microbiol. 50, 45-60. doi: 10.1046/j.13652958.2003.03674.x

Burton, E. A., Oliver, T. N., and Pendergast, A. M. (2005). Abl kinases regulate actin comet tail elongation via an N-WASP-dependent pathway. Mol. Cell. Biol. 25, 8834-8843. doi: 10.1128/MCB.25.20.8834-8843.2005

Campbell-Valois, F. X., Sachse, M., Sansonetti, P. J., and Parsot, C. (2015). Escape of actively secreting Shigella flexneri from ATG8/LC3-positive vacuoles formed during cell-to-cell spread is facilitated by IcsB and VirA. MBio 6, e02567e02514. doi: 10.1128/mBio.02567-14

Campbell-Valois, F. X., Schnupf, P., Nigro, G., Sachse, M., Sansonetti, P. J., and Parsot, C. (2014). A fluorescent reporter reveals on/off regulation of the Shigella type III secretion apparatus during entry and cell-to-cell spread. Cell Host Microbe 15, 177-189. doi: 10.1016/j.chom.2014.01.005

Carpenter, C. D., Cooley, B. J., Needham, B. D., Fisher, C. R., Trent, M. S., Gordon, $\mathrm{V}$., et al. (2014). The Vps/VacJ ABC transporter is required for intercellular spread of Shigella flexneri. Infect. Immun. 82, 660-669. doi: 10.1128/IAI.01 057-13

Charles, M., Pérez, M., Kobil, J. H., and Goldberg, M. B. (2001). Polar targeting of shigella virulence factor IcsA in enterobacteriacae and vibrio. Proc. Natl. Acad. Sci. U.S.A. 98, 9871-9876. doi: 10.1073/pnas.171310498

Dong, N., Zhu, Y., Lu, Q., Hu, L., Zheng, Y., and Shao, F. (2012). Structurally distinct bacterial TBC-like GAPs link Arf GTPase to Rab1 inactivation to counteract host defenses. Cell 150, 1029-1041. doi: 10.1016/j.cell.2012. 06.050

Dragoi, A. M., and Agaisse, H. (2014). The serine/threonine kinase STK11 promotes Shigella flexneri dissemination through establishment of cell-cell contacts competent for tyrosine kinase signaling. Infect. Immun. 82, 4447-4457. doi: 10.1128/IAI.02078-14

Dragoi, A. M., and Agaisse, H. (2015). The Class II phosphatidylinositol-3 phosphate kinase PIK3C2A promotes Shigella flexneri dissemination through formation of vacuole-like protrusions. Infect. Immun. 83, 1695-1704. doi: 10.1128/IAI.03138-14

Dragoi, A. M., Talman, A. M., and Agaisse, H. (2013). Bruton's tyrosine kinase regulates Shigella flexneri dissemination in HT-29 intestinal cells. Infect. Immun. 81, 598-607. doi: 10.1128/IAI.00853-12
Egile, C., Loisel, T. P., Laurent, V., Li, R., Pantaloni, D., Sansonetti, P. J., et al. (1999). Activation of the CDC42 effector N-WASP by the Shigella flexneri IcsA protein promotes actin nucleation by Arp2/3 complex and bacterial actin-based motility. J. Cell Biol. 146, 1319-1332. doi: 10.1083/jcb.146.6.1319

Fixen, K. R., Janakiraman, A., Garrity, S., Slade, D. J., Gray, A. N., Karahan, N., et al. (2012). Genetic reporter system for positioning of proteins at the bacterial pole. MBio 3:e00251-11. doi: 10.1128/mBio.00251-11

Fukumatsu, M., Ogawa, M., Arakawa, S., Suzuki, M., Nakayama, K., Shimizu, S., et al. (2012). Shigella targets epithelial tricellular junctions and uses a noncanonical clathrin-dependent endocytic pathway to spread between cells. Cell Host Microbe 11, 325-336. doi: 10.1016/j.chom.2012.03.001

Goldberg, M. B., Barzu, O., Parsot, C., and Sansonetti, P. J. (1993). Unipolar localization and ATPase activity of IcsA, a Shigella flexneri protein involved in intracellular movement. Infect. Agents Dis. 2, 210-211.

Gouin, E., Gantelet, H., Egile, C., Lasa, I., Ohayon, H., Villiers, V., et al. (1999) A comparative study of the actin-based motilities of the pathogenic bacteria Listeria monocytogenes, Shigella flexneri and Rickettsia conorii. J. Cell. Sci. 112(Pt 11), 1697-1708.

Heindl, J. E., Saran, I., Yi, C. R., Lesser, C. F., and Goldberg, M. B. (2010). Requirement for formin-induced actin polymerization during spread of Shigella flexneri. Infect. Immun. 78, 193-203. doi: 10.1128/IAI.00252-09

Henderson, I. R., and Nataro, J. P. (2005). Autotransporter proteins. Ecosal Plus 1. doi: 10.1128/ecosal.8.7.3

Ho, H. Y., Rohatgi, R., Lebensohn, A. M., Le, M., Li, J., Gygi, S. P., et al. (2004). Toca-1 mediates Cdc42-dependent actin nucleation by activating the N-WASP-WIP complex. Cell 118, 203-216. doi: 10.1016/j.cell.2004.06.027

Hong, M., Gleason, Y., Wyckoff, E. E., and Payne, S. M. (1998). Identification of two Shigella flexneri chromosomal loci involved in intercellular spreading. Infect. Immun. 66, 4700-4710.

d'Hauteville, H., and Sansonetti, P. J. (1992). Phosphorylation of IcsA by cAMPdependent protein kinase and its effect on intracellular spread of Shigella flexneri. Mol. Microbiol. 6, 833-841. doi: 10.1111/j.1365-2958.1992.tb01534.x

Kadurugamuwa, J. L., Rohde, M., Wehland, J., and Timmis, K. N. (1991). Intercellular spread of Shigella flexneri through a monolayer mediated by membranous protrusions and associated with reorganization of the cytoskeletal protein vinculin. Infect. Immun. 59, 3463-3471.

Kane, C. D., Schuch, R., Day, W. A. Jr., and Maurelli, A. T. (2002). MxiE regulates intracellular expression of factors secreted by the Shigella flexneri 2a type III secretion system. J. Bacteriol. 184, 4409-4419. doi: 10.1128/JB.184.16.44094419.2002

Kim, A. S., Kakalis, L. T., Abdul-Manan, N., Liu, G. A., and Rosen, M. K. (2000). Autoinhibition and activation mechanisms of the Wiskott-Aldrich syndrome protein. Nature 404, 151-158. doi: 10.1038/35004513

Kim, M., Ogawa, M., Fujita, Y., Yoshikawa, Y., Nagai, T., Koyama, T., et al. (2009). Bacteria hijack integrin-linked kinase to stabilize focal adhesions and block cell detachment. Nature 459, 578-582. doi: 10.1038/nature07952

Kreibich, S., Emmenlauer, M., Fredlund, J., Rämö, P., Münz, C., Dehio, C., et al. (2015). Autophagy proteins promote repair of endosomal membranes damaged by the salmonella type three secretion system 1. Cell Host Microbe 18, 527-537. doi: 10.1016/j.chom.2015.10.015

Kuehl, C. J., Dragoi, A. M., and Agaisse, H. (2014). The Shigella flexneri type 3 secretion system is required for tyrosine kinase-dependent protrusion resolution, and vacuole escape during bacterial dissemination. PLoS ONE 9:e112738. doi: 10.1371/journal.pone.0112738

Kuehl, C. J., Dragoi, A. M., Talman, A., and Agaisse, H. (2015). Bacterial spread from cell to cell: beyond actin-based motility. Trends Microbiol. 23, 558-566. doi: 10.1016/j.tim.2015.04.010

Leung, Y., Ally, S., and Goldberg, M. B. (2008). Bacterial actin assembly requires toca-1 to relieve N-wasp autoinhibition. Cell Host Microbe 3, 39-47. doi: 10.1016/j.chom.2007.10.011

Loisel, T. P., Boujemaa, R., Pantaloni, D., and Carlier, M. F. (1999). Reconstitution of actin-based motility of Listeria and Shigella using pure proteins. Nature 401 613-616. doi: 10.1038/44183

Lum, M., and Morona, R. (2014). Myosin IIA is essential for Shigella flexneri cell-to-cell spread. Pathog Dis. 72, 174-187. doi: 10.1111/2049-632X.12202

Mac Síomóin, R. A., Nakata, N., Murai, T., Yoshikawa, M., Tsuji, H., and Sasakawa, C. (1996). Identification and characterization of ispA, a Shigella flexneri chromosomal gene essential for normal in vivo cell division and 
intracellular spreading. Mol. Microbiol. 19, 599-609. doi: 10.1046/j.13652958.1996.405941.x

Makino, S., Sasakawa, C., Kamata, K., Kurata, T., and Yoshikawa, M. (1986). A genetic determinant required for continuous reinfection of adjacent cells on large plasmid in S. flexneri 2a. Cell 46, 551-555. doi: 10.1016/00928674(86)90880-9

Malinverni, J. C., and Silhavy, T. J. (2009). An ABC transport system that maintains lipid asymmetry in the gram-negative outer membrane. Proc. Natl. Acad. Sci. U.S.A. 106, 8009-8014. doi: 10.1073/pnas.0903229106

Mavris, M., Page, A. L., Tournebize, R., Demers, B., Sansonetti, P., and Parsot, C. (2002). Regulation of transcription by the activity of the Shigella flexneri type III secretion apparatus. Mol. Microbiol. 43, 1543-1553. doi: 10.1046/j.13652958.2002.02836.x

May, K. L., and Morona, R. (2008). Mutagenesis of the Shigella flexneri autotransporter IcsA reveals novel functional regions involved in IcsA biogenesis and recruitment of host neural Wiscott-Aldrich syndrome protein. J. Bacteriol. 190, 4666-4676. doi: 10.1128/JB.00093-08

Mellouk, N., Weiner, A., Aulner, N., Schmitt, C., Elbaum, M., Shorte, S. L., et al. (2014). Shigella subverts the host recycling compartment to rupture its vacuole. Cell Host Microbe 16, 517-530. doi: 10.1016/j.chom.2014.09.005

Mimuro, H., Suzuki, T., Suetsugu, S., Miki, H., Takenawa, T., and Sasakawa, C. (2000). Profilin is required for sustaining efficient intra- and intercellular spreading of Shigella flexneri. J. Biol. Chem. 275, 28893-28901. doi: 10.1074/jbc.M003882200

Miura, M., Terajima, J., Izumiya, H., Mitobe, J., Komano, T., and Watanabe, H. (2006). OspE2 of Shigella sonnei is required for the maintenance of cell architecture of bacterium-infected cells. Infect. Immun. 74, 2587-2595. doi: 10.1128/IAI.74.5.2587-2595.2006

Mogull, S. A., Runyen-Janecky, L. J., Hong, M., and Payne, S. M. (2001). dksA is required for intercellular spread of Shigella flexneri via an RpoS-independent mechanism. Infect. Immun. 69, 5742-5751. doi: 10.1128/IAI.69.9.57425751.2001

Morona, R., L., van den Bosch, and Manning, P. A. (1995). Molecular, genetic, and topological characterization of $\mathrm{O}$-antigen chain length regulation in Shigella flexneri. J. Bacteriol. 177, 1059-1068.

Mounier, J., Laurent, V., Hall, A., Fort, P., Carlier, M. F., Sansonetti, P. J., et al. (1999). Rho family GTPases control entry of Shigella flexneri into epithelial cells but not intracellular motility. J. Cell. Sci. 112(Pt 13), 2069-2080.

Musher, D. M., and Musher, B. L. (2004). Contagious acute gastrointestinal infections. N. Engl. J. Med. 351, 2417-2427. doi: 10.1056/NEJMra041837

Nakata, N., Sasakawa, C., Okada, N., Tobe, T., Fukuda, I., Suzuki, T., et al. (1992). Identification and characterization of virK, a virulence-associated large plasmid gene essential for intercellular spreading of Shigella flexneri. Mol. Microbiol. 6, 2387-2395. doi: 10.1111/j.1365-2958.1992.tb01413.x

Nakata, N., Tobe, T., Fukuda, I., Suzuki, T., Komatsu, K., Yoshikawa, M., et al. (1993). The absence of a surface protease, OmpT, determines the intercellular spreading ability of Shigella: the relationship between the ompT and kcpA loci. Mol. Microbiol. 9, 459-468. doi: 10.1111/j.1365-2958.1993.tb 01707.x

Oaks, E. V., Wingfield, M. E., and Formal, S. B. (1985). Plaque formation by virulent Shigella flexneri. Infect. Immun. 48, 124-129.

Ogawa, M., Suzuki, T., Tatsuno, I., Abe, H., and Sasakawa, C. (2003). IcsB, secreted via the type III secretion system, is chaperoned by IpgA and required at the post-invasion stage of Shigella pathogenicity. Mol. Microbiol. 48, 913-931. doi: 10.1046/j.1365-2958.2003.03489.x

Ogawa, M., Yoshimori, T., Suzuki, T., Sagara, H., Mizushima, N., and Sasakawa, C. (2005). Escape of intracellular Shigella from autophagy. Science 307, 727-731. doi: $10.1126 /$ science. 1106036

Okamura, N., and Nakaya, R. (1977). Rough mutant of Shigella flexneri 2a that penetrates tissue culture cells but does not evoke keratoconjunctivitis in guinea pigs. Infect. Immun. 17, 4-8.

Page, A. L., Ohayon, H., Sansonetti, P. J., and Parsot, C. (1999). The secreted $\mathrm{IpaB}$ and $\mathrm{IpaC}$ invasins and their cytoplasmic chaperone $\mathrm{IpgC}$ are required for intercellular dissemination of Shigella flexneri. Cell. Microbiol. 1, 183-193. doi: 10.1046/j.1462-5822.1999.00019.x

Parsot, C., Ageron, E., Penno, C., Mavris, M., Jamoussi, K., d'Hauteville, H., et al. (2005). A secreted anti-activator, OspD1, and its chaperone, Spa15, are involved in the control of transcription by the type III secretion apparatus activity in Shigella flexneri. Mol. Microbiol. 56, 1627-1635. doi: 10.1111/j.13652958.2005.04645.x

Purdy, G. E., Fisher, C. R., and Payne, S. M. (2007). IcsA surface presentation in Shigella flexneri requires the periplasmic chaperones DegP, Skp, and SurA. J. Bacteriol. 189, 5566-5573. doi: 10.1128/JB.00483-07

Purdy, G. E., Hong, M., and Payne, S. M. (2002). Shigella flexneri DegP facilitates IcsA surface expression and is required for efficient intercellular spread. Infect. Immun. 70, 6355-6364. doi: 10.1128/IAI.70.11.6355-6364.2002

Rajakumar, K., Jost, B. H., Sasakawa, C., Okada, N., Yoshikawa, M., and Adler, B. (1994). Nucleotide sequence of the rhamnose biosynthetic operon of Shigella flexneri $2 \mathrm{a}$ and role of lipopolysaccharide in virulence. J. Bacteriol. 176, 2362-2373.

Rathman, M., de Lanerolle, P., Ohayon, H., Gounon, P., and Sansonetti, P. (2000a). Myosin light chain kinase plays an essential role in S. flexneri dissemination. J. Cell Sci. 113(Pt 19), 3375-3386.

Rathman, M., Jouirhi, N., Allaoui, A., Sansonetti, P., Parsot, C., and Tran Van Nhieu G. (2000b). The development of a FACS-based strategy for the isolation of Shigella flexneri mutants that are deficient in intercellular spread. Mol. Microbiol. 35, 974-990. doi: 10.1046/j.1365-2958.2000.01770.x

Rohatgi, R., Ma, L., Miki, H., Lopez, M., Kirchhausen, T., Takenawa, T., et al. (1999). The interaction between N-WASP and the Arp2/3 complex links Cdc42-dependent signals to actin assembly. Cell 97, 221-231. doi: 10.1016/S0092-8674(00)80732-1

Runyen-Janecky, L. J., Boyle, A. M., Kizzee, A., Liefer, L., and Payne, S. M. (2005). Role of the Pst system in plaque formation by the intracellular pathogen Shigella flexneri. Infect. Immun. 73, 1404-1410. doi: 10.1128/IAI.73.3.1404-1410.2005

Runyen-Janecky, L. J., and Payne, S. M. (2002). Identification of chromosomal Shigella flexneri genes induced by the eukaryotic intracellular environment. Infect. Immun. 70, 4379-4388. doi: 10.1128/IAI.70.8.4379-4388.2002

Sandlin, R. C., Lampel, K. A., Keasler, S. P., Goldberg, M. B., Stolzer, A. L., and Maurelli, A. T. (1995). Avirulence of rough mutants of Shigella flexneri: requirement of $\mathrm{O}$ antigen for correct unipolar localization of IcsA in the bacterial outer membrane. Infect. Immun. 63, 229-237.

Sansonetti, P. J. (1998). Molecular and cellular mechanisms of invasion of the intestinal barrier by enteric pathogens. the paradigm of Shigella. Folia Microbiol. (Praha) 43, 239-46. doi: 10.1007/BF02818608

Sansonetti, P. J., Mounier, J., Prévost, M. C., and Mège, R. M. (1994). Cadherin expression is required for the spread of Shigella flexneri between epithelial cells. Cell 76, 829-839. doi: 10.1016/0092-8674(94)90358-1

Santapaola, D., Del Chierico, F., Petrucca, A., Uzzau, S., Casalino, M., Colonna, B., et al. (2006). Apyrase, the product of the virulence plasmid-encoded phoN2 (apy) gene of Shigella flexneri, is necessary for proper unipolar IcsA localization and for efficient intercellular spread. J. Bacteriol. 188, 1620-1627. doi: 10.1128/JB.188.4.1620-1627.2006

Schuch, R., Sandlin, R. C., and Maurelli, A. T. (1999). A system for identifying post-invasion functions of invasion genes: requirements for the Mxi-Spa type III secretion pathway of Shigella flexneri in intercellular dissemination. Mol. Microbiol. 34, 675-689. doi: 10.1046/j.1365-2958.1999.01627.x

Scribano, D., Petrucca, A., Pompili, M., Ambrosi, C., Bruni, E., Zagaglia, C., et al. (2014). Polar localization of PhoN2, a periplasmic virulence-associated factor of Shigella flexneri, is required for proper IcsA exposition at the old bacterial pole. PLoS ONE 9:e90230. doi: 10.1371/journal.pone.0090230

Sharma, A. K., and Payne, S. M. (2006). Induction of expression of hfq by DksA is essential for Shigella flexneri virulence. Mol. Microbiol. 62, 469-479. doi: 10.1111/j.1365-2958.2006.05376.x

Shere, K. D., Sallustio, S., Manessis, A., D’Aversa, T. G., and Goldberg, M. B. (1997). Disruption of IcsP, the major Shigella protease that cleaves IcsA, accelerates actin-based motility. Mol. Microbiol. 25, 451-462. doi: 10.1046/j.1365-2958.1997.4681827.x

Shibata, T., Takeshima, F., Chen, F., Alt, F. W., and Snapper, S. B. (2002). Cdc42 facilitates invasion but not the actin-based motility of Shigella. Curr. Biol. 12 341-345. doi: 10.1016/S0960-9822(02)00689-9

Sidik, S., Kottwitz, H., Benjamin, J., Ryu, J., Jarrar, A., Garduno, R., et al. (2014). A Shigella flexneri virulence plasmid encoded factor controls production of outer membrane vesicles. G3 (Bethesda) 4, 2493-2503. doi: 10.1534/g3.114.014381

Suzuki, T., Miki, H., Takenawa, T., and Sasakawa, C. (1998). Neural WiskottAldrich syndrome protein is implicated in the actin-based motility of Shigella flexneri. EMBO J. 17, 2767-2776. doi: 10.1093/emboj/17.10.2767 
Suzuki, T., Mimuro, H., Suetsugu, S., Miki, H., Takenawa, T., and Sasakawa, C. (2002). Neural Wiskott-Aldrich syndrome protein (N-WASP) is the specific ligand for Shigella VirG among the WASP family and determines the host cell type allowing actin-based spreading. Cell. Microbiol. 4, 223-233. doi: 10.1046/j.1462-5822.2002.00185.x

Suzuki, T., Murai, T., Fukuda, I., Tobe, T., Yoshikawa, M., and Sasakawa, C. (1994). Identification and characterization of a chromosomal virulence gene, vacJ, required for intercellular spreading of Shigella flexneri. Mol. Microbiol. 11, 31-41. doi: 10.1111/j.1365-2958.1994.tb00287.x

Suzuki, T., Saga, S., and Sasakawa, C. (1996). Functional analysis of Shigella VirG domains essential for interaction with vinculin and actin-based motility. J. Biol. Chem. 271, 21878-21885. doi: 10.1074/jbc.271.36.21878

Takeuchi, A., Formal, S. B., and Sprinz, H. (1968). Experimental acute colitis in the Rhesus monkey following peroral infection with Shigella flexneri. An electron microscope study. Am. J. Pathol. 52, 503-529.

Talman, A. M., Chong, R., Chia, J., Svitkina, T., and Agaisse, H. (2014). Actin network disassembly powers dissemination of Listeria monocytogenes. J. Cell Sci. 127, 240-249. doi: $10.1242 /$ jcs. 140038

Tran Van Nhieu, G., Clair, C., Bruzzone, R., Mesnil, M., Sansonetti, P., and Combettes, L. (2003). Connexin-dependent inter-cellular communication increases invasion and dissemination of Shigella in epithelial cells. Nat. Cell Biol. 5, 720-726. doi: $10.1038 /$ ncb 1021

Uchiya, K., Tobe, T., Komatsu, K., Suzuki, T., Watarai, M., Fukuda, I., et al. (1995). Identification of a novel virulence gene, virA, on the large plasmid of Shigella, involved in invasion and intercellular spreading. Mol. Microbiol. 17, 241-250. doi: 10.1111/j.1365-2958.1995.mmi_17020241.x

Van den Bosch, L., Manning, P. A., and Morona, R. (1997). Regulation of Oantigen chain length is required for Shigella flexneri virulence. Mol. Microbiol. 23, 765-775. doi: 10.1046/j.1365-2958.1997.2541625.x
Vasselon, T., Mounier, J., Hellio, R., and Sansonetti, P. J. (1992). Movement along actin filaments of the perijunctional area and de novo polymerization of cellular actin are required for Shigella flexneri colonization of epithelial Caco-2 cell monolayers. Infect. Immun. 60, 1031-1040.

Veiga, E., and Cossart, P. (2006). The role of clathrin-dependent endocytosis in bacterial internalization. Trends Cell Biol. 16, 499-504. doi: 10.1016/j.tcb.2006.08.005

Watarai, M., Tobe, T., Yoshikawa, M., and Sasakawa, C. (1995). Disulfide oxidoreductase activity of Shigella flexneri is required for release of Ipa proteins and invasion of epithelial cells. Proc. Natl. Acad. Sci. U.S.A. 92, 4927-4931. doi: 10.1073/pnas.92.11.4927

Welch, M. D., and Way, M. (2013). Arp2/3-mediated actin-based motility: a tail of pathogen abuse. Cell Host Microbe 14, 242-255. doi: 10.1016/j.chom.2013.08.011

Yu, J., Edwards-Jones, B., Neyrolles, O., and Kroll, J. S. (2000). Key role for DsbA in cell-to-cell spread of Shigella flexneri, permitting secretion of Ipa proteins into interepithelial protrusions. Infect. Immun. 68, 6449-6456. doi: 10.1128/IAI.68.11.6449-6456.2000

Conflict of Interest Statement: The author declares that the research was conducted in the absence of any commercial or financial relationships that could be construed as a potential conflict of interest.

Copyright () 2016 Agaisse. This is an open-access article distributed under the terms of the Creative Commons Attribution License (CC BY). The use, distribution or reproduction in other forums is permitted, provided the original author(s) or licensor are credited and that the original publication in this journal is cited, in accordance with accepted academic practice. No use, distribution or reproduction is permitted which does not comply with these terms. 